\title{
VARIATIONS OF PHYSICAL QUANTITIES UNDER BMS TRANSFORMATIONS
}

\author{
(Presented by J. Einasto)
}

\section{Introduction}

Since the discovery that at null infinity asymptotically flat spacetimes possess a symmetry group larger than the Poincaré group $\left[{ }^{1,2}\right]$, now called the BMS (Bondi-Metzner-Sachs) group, this group has aroused two different attitudes. One may either reduce it to the Poincaré group or obtain physical consequences of namely BMS symmetries. The first possibility has been consistently realized almost twenty years later with Penrose's twistor formalism, now a flourishing branch of the gravitation theory with its own journal "Twistor Newsletters". (See e.g. $\left.{ }^{3}\right]$ and references therein.) As to the second possibility, some nice consequences of the BMS symmetry have been also found [ $\left.{ }^{4}\right]$, but an objection to such kind of activities lies in the disturbing fact that angular momentum depends on supertranslations. (The latter stands for extra BMS symmetries and is probably misleading, as it originates from the presupersymmetric era.)

The aim of the present paper is not insomuch to apologize for the BMS symmetry, as to investigate the possibility of overcoming the objection mentioned. In Sec. 2, we shall examine to what extent the variations of physical quantities under Poincaré transformations correspond to expectations. Then, in Sec. 3, we shall look for conditions in which the variation of angular momentum under supertranslations may be understood or even eliminated. To carry out the calculations, we shall first recall the necessary formulae from our previous paper $\left[{ }^{5}\right]$, writing them in a compact form suitable for their further use. This formalism, described in Sec. 1, is rather promising and may have independent applications.

\section{Variations of Bondi integration functions}

The Bondi integration functions $M(u, \theta, \Phi), N(u, \theta, \Phi)$ and $c(u, \theta, \Phi)$ are introduced when the Einstein equations are integrated in the Bondi coordinates $\left.{ }^{1}\right]$. (Here $u$ stands for the retarded time coordinate, $\theta$ and $\Phi$ are ordinary angle variables on a sphere; the integration has been carried out with respect to the fourth coordinate, the radial variable $r$.) The physical meaning of $M$ and $N$ relies on their monopole and dipole parts defining the physical quantities we are interested in, viz the total 
mass $m$, momentum $p^{j}$, angular momentum $l^{j}$ and dipole momentum $d^{j}$ as follows:

$$
\begin{aligned}
& M(u, \theta, \Phi)=m(u)+3 p^{j}(u) y_{j}(\theta, \Phi)+\ldots \\
& N(u, \theta, \Phi)=\left(d^{j}(u)-\mathrm{i} l^{j}(u)\right) \tilde{\partial} y_{j}(\theta, \Phi)+\ldots
\end{aligned}
$$

Here we have used a vector function on a sphere

$$
y_{j}=(\sin \theta \cos \Phi, \sin \theta \sin \Phi, \cos \theta)
$$

instead of spherical harmonics $Y_{1 m}$ (and $\tilde{\partial} y$ instead of spin spherical harmonics ${ }_{-1} Y_{1 \mathrm{~m}}$ ) inspired by more distinct appearance of the formulae, as well as for the convenience of further calculations. Some useful formulae related to $y$ are given in Appendix B.

In our previous paper $\left[^{4}\right]$, we obtained the variations of $M, N$ and $c$ under the BMS transformations and showed that they form a representation of the group. Here we shall reveal the possibility of combining them into one formula. First we recall that an infinitesimal BMS transformation may be expressed through two functions, $\tau(u, \theta, \Phi)$ and $\omega(\theta, \Phi)$, which are introduced with infinitesimal asymptotic coordinate transformations

$$
\begin{aligned}
& \delta u=\tau(u, \theta, \Phi), \\
& \delta \theta-i \sin \theta \delta \Phi=\omega(\theta, \Phi)+O(1 / r) .
\end{aligned}
$$

These functions will form Lie algebra of the BMS group if they are restricted by the conditions

$$
\begin{gathered}
\partial \omega+\tilde{\partial} \bar{\omega}=-4 \dot{\tau}, \\
\dot{\omega}=\tilde{\partial}_{\omega}=0 .
\end{gathered}
$$

(Here and later on, a dot over a function denotes a derivative with respect to $u$. The necessary knowledge about the edth operators $\partial, \tilde{\partial}$ may be found in Appendix A.) The BMS representation related to the transformation of $M, N$ and $c$ (the formula (3.6) in [5]) may be written as

$$
\delta_{\tau, \omega} F_{s}=R_{3, s}(\tau, \omega) F_{s}-(\tilde{\partial} \tau) F_{s+1}, s=-1,0,1,2 .
$$

Here $F_{s}(u, \theta, \Phi)$, functions with spin weight $s$, denote the following combinations of $N, M$ and $c$ :

$F_{-1}=N, \quad F_{0}=M+c \dot{\bar{c}}+\frac{1}{4}(\tilde{\partial} \tilde{\bar{c}} \bar{c}-\partial \partial c), \quad F_{1}=\tilde{\partial} \dot{\bar{c}}, \quad F_{2}=\ddot{\bar{c}}, \quad F_{3}=0$

and $R_{v, s}(\tau, \omega)$ stands for the spin and boost weighed operator,

$$
R_{v, s}(\tau, \omega)=-\tau \frac{\partial}{\partial u}+\frac{1}{2} \omega \partial_{s}+\frac{1}{2}-\tilde{\partial}_{s}-\dot{v} \dot{\tau}+\frac{s}{4}(\partial \omega-\tilde{\partial} \bar{\omega}),
$$

$R_{0,0}$ acting as the BMS generator on a scalar function.

Functions $F_{s}$ are not independent, they satisfy

$$
(2-s) \dot{F}_{s}=\tilde{\partial} F_{s+1}+2 c F_{s+2} \text {. }
$$

For $s=-1,0$, this equation is a direct consequence of the so-called supplementary conditions on $N$ and $M$,

$$
\begin{aligned}
3 \dot{N} & =\tilde{\partial} M+(\tilde{\partial} c) \dot{\bar{c}}+3 c \tilde{\partial} \dot{\bar{c}}+\frac{1}{4} \tilde{\partial}(\tilde{\partial} \tilde{\partial} \bar{c}-\partial \partial c), \\
\dot{M} & =-\dot{\dot{c}} \dot{\bar{c}}+\frac{1}{4}(ð \partial \dot{c}+\tilde{\partial} \tilde{\bar{\partial}}),
\end{aligned}
$$


for $s=1$, it expresses simply the interdependence between $\vec{F}_{1}$ and $\vec{F}_{2}$ due to their definition (1.6).

The compact form of the formulae enables an easy proof of the consistency of BMS transformations (1.5) with supplementary conditions $(1.8)$, i. e. the validity of the equation $\delta\left(\hat{F}_{s}\right)=\left(\delta F_{s}\right)$. To verify it, one has to use the transformation rule for $c$,

$$
\delta_{\tau, \omega} c=R_{1,-2}(\tau, \omega) c+\frac{1}{2} \tilde{\delta} \tilde{\delta} \tau
$$

and an identity

$$
R_{v+1, s-1}(\tau, \omega) \tilde{\delta}_{s}-\tilde{\partial}_{s} R_{v, s}(\tau, \omega)=(\tilde{\partial} \tau) \frac{\partial}{\partial u}+(v-s)(\tilde{\partial} \dot{\tau}),
$$

deducible from the definition of $R_{v, s}(\tau, \omega)$ (1.7) with the help of restrictions (1.4) on $\tau$ and $\omega$.

Finally, we rewrite (1.5) with the help of (1.8) in the form

$$
\delta_{\tau, \omega} F_{s}=\widetilde{R}_{3, s}(\tau, \omega) F_{s}-\frac{\tau}{2+s} \tilde{\delta} F_{s+1}-(\tilde{\partial} \tau) F_{s+1}-\frac{2 \tau}{2+s} c F_{s+2},
$$

where

$$
\widetilde{R}_{v, s}=\frac{1}{2} \omega \tilde{\partial}_{s}+\frac{1}{2} \bar{\omega} \tilde{\partial}_{s}-v \dot{\tau}+\frac{s}{4}(\tilde{\omega}-\tilde{\partial} \bar{\omega}) .
$$

This form of the formula will be more suitable for studying the properties of the variations.

\section{Variations of physical quantities under Poincaré transformations}

For a concrete transformation, we must specify the form of functions $\tau$ and $\omega$. As to the infinitesimal Poincaré transformations, $\tau$ and $\omega$ may be written with the help of convention (1.2) as follows:

The proper Lorentz-transformation by $\delta v^{k}$ along $k$-axis

$$
\tau=u y_{k} \delta v^{k}, \quad \omega=\tilde{\delta} y_{k} \delta v^{k} .
$$

The 3-rotation by $ð \alpha^{k}$ around $k$-axis

$$
\tau=0, \quad \omega=-\mathrm{i} \tilde{\partial} y_{k} \delta \alpha^{k} .
$$

The translation by $\delta x^{k}$ along $k$-axis

$$
\tau=y_{k} \delta x^{k}, \quad \omega=0 .
$$

The time translation by $\delta t$

$$
\tau=\delta t, \quad \omega=0 .
$$

Substituting $\tau, \omega$ for $(2.1)$ to (2.4) in (1.11) and taking (1.6), (1.1) into account, we obtain the desired variations of physical quantities. In fact, the quantities we are interested in are the conserved quantities corresponding to Poincaré symmetries. Certainly, they are conserved only in a closed system, but we are considering a radiative solution with monotonous changes. We shall leave the study of effects caused by radiation towards the end of the section and, for the time being, neglect the part of variations connected with monotonotis changes, i. e. we shall first 
ignore the terms quadratic in the information function $c$, their exact form being

$$
\delta_{\tau, \omega}^{2} M=\tau \dot{\bar{c}} \dot{\bar{c}}, \quad \delta_{\tau, \omega}^{2} N=-\tau c \tilde{\widetilde{\partial}} \dot{\bar{c}}-\frac{1}{3} \tau(\tilde{\tilde{\delta}} c) \dot{\bar{c}}-(\tilde{\delta} \tau) c \dot{\bar{c}} .
$$

Now, instead of (1.11), we are left with

$$
\delta_{\tau, \omega} F_{s}=\widetilde{R}_{3, s}(\tau, \omega) F_{s}-\frac{\tau}{2+s} \tilde{\partial} F_{s+1}-(\tilde{\partial} \tau) F_{s+1} .
$$

Simultaneously, up to dipole terms, we may identify $F_{0}$ with $M$, thus, the monopole and dipole parts of the left side of the formula (2.6) describe the variations of physical quantities defined by (1.1).

To start with $(2.6)$, we recall that the multiplication of multipoles obeys the «triangular law», hence, if $\tau$, $\omega$ have only monopole and dipole parts as in (2.1)-(2.4), we shall obtain from (2.6) that the change in $2^{l}$-pole part of $F_{s}$ depends only on $2^{l-1}, 2^{l}$ and $2^{l+1}$-pole parts of $F_{s}$ and $F_{s+1}$. In the case of a finite transformation, generically all multipoles affect each other, but in special cases this affection may be cut off in some place. For (2.6) it is just the case, and we express the outcome as a theorem.

Theorem. If functions $F_{s}$ transform according to (2.6) with $\tau$, $\omega$ taking values from $(2.1)-(2.4)$ then the monopole and dipole parts of the $F_{s}$ will transform independently of higher multipoles.

The theorem follows directly from two lemmas proved in Appendix C if we take $v=3$ in lemma 1 and $v=2$ in lemma 2 .

Due to the theorem, we may conclude that when the expansion series transform according to (2.6), the variations of the physical quantities in (1.1) depend only on each other, any other knowledge of higher multipoles is not needed. (The theorem still allows the dependence of $d^{k}$ and $l^{k}$ on dipole part of $F_{1}=\tilde{\partial} \tilde{\bar{c}}$, but it does not matter, as the latter vanishes identically.) The actual expression for these variations are calculated in Appendix D; they certainly do not need any explanation, except perhaps (D.5), the variation of angular momentum under proper Lorentz transformation. Here we must take into account that in the approximation without $c^{2}$-terms $\dot{d}=p, \dot{p}=0$. Thus, we may take $d=d_{0}+u p$ and we obtain instead of (D.5)

$$
\delta l^{j}=\varepsilon_{j k l} \delta v^{k} d^{l},
$$

a completely expected result if we recall that $d^{j}$ can be interpreted as the cartesian coordinates of the centre of mass location multiplied by the mass of the system.

So we have presented a thorough treatment of variations described by (2.6). The same cannot be done with the remaining part of variations (2.5), nevertheless, some interpretable results may be obtained. We also learn what additional knowledge would be needed for a complete treatment.

Right away we notice that (2.5) does not depend on $\omega$, hence 3-rotations (2.2) give us nothing. The effect of proper Lorentz transformations (2.1) will be determined by that of translations (2.3) if we replace $\delta x^{k}$ by $u \delta v^{k}$. Thus it suffices to consider translations (2.3) and (2.4).

Regarding the time translations (2.4), its effect is uniform for all quantities $m, p^{j}, l^{j}, d^{j}$. Let $q$ denote any of them, then

$$
\delta q=-\dot{q} \delta t .
$$


To interpret this, let us recall that in fact $q$ means the amount of corresponding quantity in a region enclosed by a two-surface at future nullinfinity. If we fix the location of this surface by Minkowskian coordinates $x, t$ then $q$ may be regarded as a function $q(x, t)$. In such a case, (2.7) expresses the equality of derivatives in respect to $t$ and $u$ except the minus sign. The latter can be explained if we recall that until now the variation has had the meaning of $\delta q(x, t)=q^{\prime}(x, t)-q(x, t)$. Thus, we shall obtain a derivative with the opposite sign in (2.7) of we assume $q^{\prime}\left(x^{\prime}, t^{\prime}\right)=$ $=q(x, t)$.

Continuing analysis of variations (2.5) with the space translations (2.3), we can see that the situation is more complicated. Only $\delta m$ is completely defined. With (B.7) we find $\delta m=-p^{k} \delta_{k j} \delta x^{j}$. Analogically with the previous case, it may be interpreted as

$$
\frac{\partial m(x, t)}{\partial x^{j}}=\dot{p}^{j}(x, t)
$$

a fairly reasonable outcome if we recall that $m$ is here the radiative energy departing with the unit velocity from the origin of coordinates.

As we reach $\delta p$, we cannot ignore quadrupole terms any more. Let.

$$
M+\frac{1}{4}(ð \check{c}+\tilde{\partial} \tilde{\bar{c}})=m+3 p^{j} y_{j}+Q_{k l}\left(y_{k} y_{l}-\frac{1}{3} \delta_{k l}\right)+\ldots
$$

then we find with (B.8)

$$
\frac{\partial p^{k}}{\partial x^{l}}=\frac{1}{3} \delta_{k l}\left\{\dot{m}-\frac{2}{15} Q_{j j}\right\}+\frac{1}{15}\left\{Q_{k l}+Q_{l k}\right\}
$$

When this equation is multiplied by $\delta_{k l}$, we find $\frac{\partial p^{k}}{\partial x^{k}}=\dot{m}$, the expression for energy conservation. To go on with the interpretation of $(2.10)$, we must first attach physical meaning to the quadrupole terms of $M$, and the same will hold if we want to understand (2.5) with respect $\operatorname{tg} \delta l$ and $\delta d$. We are not going to do it here, we merely take it as a hint for the next section that for the complete interpretation of the variations of physical quantities under Poincaré transformations, knowledge of higher multipoles is needed.

\section{Variation of angular momentum under supertranslations}

The extra freedom in the choice of $\tau, \omega$ allowed by (1.4) in addition to the Poincaré transformations (2.1) to (2.4) is such that $\tau$ may be an arbitrary function of angle variables, $\omega$ remaining zero. The monopole and dipole parts of $\tau(\theta, \Phi)$ appeared already in $(2.3),(2.4)$, the rest of $\tau$ describes new kind of transformations called supertranslations. They may be visualized as an arbitrary deformation of the closed two-surface on which physical quantities are defined. To the contrary, the ordinary translations describe rigid movements of this surface.

While analysing supertranslations, we shall consider only stationary solutions defined by the absence of radiation,

$$
\dot{c}=0 \text {, }
$$

usually supplemented by

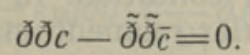


In such a case $\dot{M}=0$ as well, and the surviving transformations have the form

$$
\begin{aligned}
& \delta_{\tau} N=-\frac{1}{3} \tau(\tilde{\partial} M)-(\tilde{\partial} \tau) M, \\
& \delta_{\tau} M=0 .
\end{aligned}
$$

As a matter of fact, the effects of supertranslations and of ordinary translations occur jointly: mass $m$ and momentum $p^{k}$ are independent of either of them, whereas angular and dipole momenta depend on both. The relation between the two kinds of translations is even more intimate. If we perform a pure supertranslation and look at it from a system differing from the previous one by a finite Lorentz transformation, we observe the presence of ordinary translations as well. Hence, we cannot consider supertranslations separately from the ordinary ones. So, when we are looking for the possibility of eliminating the effect of supertranslations, we incline to do it in such a system where angular momentum is independent of translations, i.e. in a system with vanishing momentum $p^{k}$. This may be achieved only if $M=m$ is constant. The latter may be regarded as an additional requirement imposed upon the solution and it is considered in detail in [6] on the grounds that otherwise, due to (1.9), the function $N$ would have monotonous growth in time, $N=(1 / 3) u \tilde{\partial} M$. This requirement may be called «the strong stationarity condition», as it excludes even more solutions, e.g. those describing non-interactive bodies which move away with constant velocities, hence, emitting no radiation and satisfying stationarity conditions. To put it more exactly, we shall call a solution of the Einstein equations in the Bondi coordinates strongly stationary if there exists a proper Lorentz transformation converting the Bondi integration function $M$ to a constant.

Starting with $M=$ const and performing on it a finite Lorentz transformation by $v^{k}=\widetilde{v}^{k}$ th $v\left(\widetilde{v}^{k}-\right.$ a vector of unit length), we find that by integrating the corresponding infinitesimal variation law

$$
M^{\prime}\left(\theta^{\prime}, \Phi^{\prime}\right)=m\left(\operatorname{ch} v+\tilde{v}^{k} y_{k}\left(\theta^{\prime}, \Phi^{\prime}\right) \operatorname{sh} v\right)^{-3} .
$$

With (3.4), we could find the effect of any supertranslation on $l^{k}$ or $d^{k}$. Instead, with the aim of obtaining simultaneously an interpretation for the outcome, we present the supertranslation $\tau\left(\theta^{\prime}, \Phi^{\prime}\right)$ as a composition of three transformations - the Lorentz transformation by $-v^{k}$, the induced supertranslation $\tau_{0}(\theta, \Phi)$ in the system with $M=$ const ( $\tau_{0}$ also involving ordinary translations), and the inverse Lorentz transformation by $v^{k}$. As it is shown in $\left.{ }^{5}\right]$, the transformations considered form a representation of the group, and thus the effect of their composition coincides with that of the original supertranslation. Concretely we obtain

$$
\delta d^{k}=-m_{0} \delta x^{k}, \quad \delta l^{j}=-m_{0} \varepsilon_{j k l} \delta x^{k} p^{l},
$$

$\delta x^{k}$ denoting the translation in the system with $M=m_{0}=$ const induced by the original supertranslation $\tau\left(\theta^{\prime}, \Phi^{\prime}\right)$ and it may be found as the dipole part of $\tau_{0}(\theta, \Phi)$,

$$
\tau_{0}(\theta, \Phi)=\tau(\theta, \Phi) /\left(\operatorname{ch} v-\widetilde{v}^{k} y_{k}(\theta, \Phi) \operatorname{sh} v\right)
$$

These are the equations (3.5) that we regard as an explanation for the variation of angular and dipole momenta under supertranslations.

As we learned in the previous section, the radiative case has some complications in respect to the Poincaré transformations already. The outcome was complete for a stationary solution defined by (3.1) only. With regard to supertranslations, we had to demand further on a fulfilment of the strong stationary condition. In such a case, we managed to preserve the meaning of angular momentum. 


\section{Áppendix A. Edth operators}

Definitions:

$$
\begin{aligned}
& \delta_{s}=-\frac{\partial}{\partial \theta}+s \cot \theta-(\mathrm{i} / \sin \theta) \frac{\partial}{\partial \Phi} \\
& \tilde{\delta}_{s}=-\frac{\partial}{\partial \theta}-s \cot \theta+(\mathrm{i} / \sin \theta) \frac{\partial}{\partial \Phi}
\end{aligned}
$$

Here $s$ is an integer determined by the spin weight of the function on which $\partial$ or $\tilde{\partial}$ acts. The spin weights for functions used in this paper are the following: -2 for $c,-1$ for $N$ and $\omega, 0$ for $M, y$ and $\tau$. The complex conjugate of a function has the spin weight of the opposite sign. The product of two functions has spin weight equal to the sum of their spin weights. Thus, the Leibnitz rule for $\partial$ or $\tilde{\delta}$ holds:

$$
\partial_{s+s^{\prime}}(A B)=\left(\partial_{s} A\right) B+A\left(\partial_{s^{\prime}} B\right) .
$$

The operator $\partial$ raises and $\tilde{\partial}$ lowers the spin weight of a function by one. If $f_{s^{\prime}}$ denotes the $2^{l}$-pole part of a function with spin weight $s$ then

$$
\tilde{\partial} f_{s^{\prime} l}=\{s(s+1)-l(l+1)\} f_{s^{\prime} l} .
$$

\section{Appendix B. Some formulae for $y_{k}(\theta, \Phi)$}

The definition:

$$
y_{1}=\sin \theta \cos \Phi, \quad y_{2}=\sin \theta \sin \Phi, \quad y_{3}=\cos \theta .
$$

Some properties in combination with edth operators:

$$
\begin{aligned}
& \partial \partial y_{k}=\tilde{\partial} \tilde{\partial} y_{k}=0, \quad \partial \tilde{\partial} y_{k}=\tilde{\partial} \partial y_{k}=-2 y_{k} . \\
& y_{k} y_{k}=1, \quad\left(\partial y_{k}\right)\left(\tilde{\partial} y_{k}\right)=2 . \\
& y_{k}\left(\partial y_{k}\right)=y_{k}\left(\tilde{\partial} y_{k}\right)=\left(\partial y_{k}\right)\left(\partial y_{k}\right)=\left(\tilde{\partial} y_{k}\right)\left(\tilde{\partial} y_{k}\right)=0 . \\
& \varepsilon_{j k l} y_{k}\left(\tilde{\partial} y_{l}\right)=\mathrm{i}\left(\tilde{\partial} y_{j}\right), \quad \varepsilon_{j k l} y_{k}\left(\partial y_{l}\right)=-\mathrm{i}\left(\partial y_{j}\right) . \\
& \boldsymbol{\varepsilon}_{j k l}\left(\tilde{\partial} y_{k}\right)\left(\partial y_{l}\right)=2 \mathrm{i} y_{j} .
\end{aligned}
$$

Functions with a certain multipolity are gained as symmetric traceless combinations of $y_{k}$, e.g. quadrupole and octopole may be presented as

$$
f_{0,2}=y_{k} y_{l}-\frac{1}{3} \delta_{k l}, \quad f_{0,3}=y_{j} y_{k} y_{l}-\frac{1}{5} \delta_{(j k} y_{l)} .
$$

With this, instead of using Clebsch-Gordan coefficients, we find directly

$$
\begin{aligned}
& y_{j} y_{k}=\left(y_{j} y_{k}-\frac{1}{3} \delta_{j k}\right)+\frac{1}{3} \delta_{j k}, \\
& y_{j}\left(y_{k} y_{l}-\frac{1}{3} \delta_{k l}\right)=\left(y_{j} y_{k} y_{l}-\frac{1}{5} \delta_{(j k} y_{l)}\right)+\frac{1}{5} \delta_{j(k} y_{l)}-\frac{2}{15} \delta_{k l} y_{j} .
\end{aligned}
$$

With the help of edth operators, other $f_{s^{\prime}}$ are found. Then with (B.4) we obtain another useful multiplication rule

$$
y_{k} \tilde{\partial} y_{l}=\frac{i}{2} \varepsilon_{j k l} \tilde{\partial} y_{j}+\frac{1}{2} \tilde{\partial}\left(y_{k} y_{l}\right) .
$$




\section{Appendix C. Proof of two lemmás}

Let $f_{s}$ be an arbitrary function with spin weight $s$ and let $f_{s, l}$ denote its $2^{l}$-pole part. In what follows, $\tau$ and $\omega$ take values from (2.1) to (2.4).

Le m m a 1. If $\delta_{\tau, \omega} f_{s}=\widetilde{R}_{v, s}(\tau, \omega) f_{s}$ then $f_{s, v-2}$ will transform independently of $f_{s, v-1}$.

Proof. The case of translations (2.3), (2.4) is trivial due to $\widetilde{R}_{v, s}(y, 0)=\widetilde{R}_{v, s}(1,0)=0$. Let us consider 3 -rotations $(2.2)$. When $s=0$ we find with $(1.3) \widetilde{R}_{v, 0}(0, \omega)=\delta \theta \frac{\partial}{\partial \theta}-\delta \Phi \frac{\partial}{\partial \Phi}$, i. e. the ordinary generator for a scalar function on a sphere which transforms each $2^{l}$-pole $f_{0, l}$ to a linear combination of $2^{l}$-poles, say $X f_{0, l}$, by the very meaning of a multipole expansion:

$$
\widetilde{R}_{v, 0}(0, \omega) f_{0, l}=X f_{0, l} .
$$

Each $f_{s, l}$ may be gained from $f_{0, l}$ with the help of edth operators. It may be checked with (1.12) that

$$
\partial \widetilde{R}_{v, s}(0, \omega) f_{s, l}=\widetilde{R}_{v, s+1}(0, \omega) ð f_{s, l}
$$

and analogically for $\tilde{\delta}$, so we obtain for each $s$

$$
\widetilde{R}_{v, s}(0, \omega) f_{s, l}=X f_{s, l} \text {. }
$$

It remains to prove the lemma for proper Lorentz transformations (2.1),

$$
\widetilde{R}_{v, s}(u y, \tilde{\partial} y)=\frac{1}{2}(\tilde{\partial} y) \partial+\frac{1}{2}(\partial y) \tilde{\partial}-v y .
$$

Denoting

$$
y f_{s, l}=X-f_{s, l-1}+X^{0} f_{s, l}+X^{+} f_{s, l+1}
$$

and using (A.2), we find

$$
\begin{gathered}
\{(\tilde{\partial} y) \partial+(\partial y) \tilde{\partial}\} f_{s, l}=\tilde{\partial} \partial\left(y f_{s, l}\right)-(\tilde{\partial} \partial y) f_{s, l}-y\left(\tilde{\partial} \partial f_{s, l}\right)= \\
=(2 l+2) X^{-} f_{s, l-1}+2 X^{0} f_{s, l}-2 l X^{+}+f_{s, l+1} .
\end{gathered}
$$

Thus with (C.3) we obtain

$\widetilde{R}_{v, s}(u y, \tilde{\partial} y) f_{s, l}=(l+1-v) X^{-} f_{s, l-1}+(1-v) X^{0} f_{s, l}-(l+v) X+f_{s, l+1}$.

The factor $(l+1-v)$ in front of $f_{s, l-1}$ completes the proof of the lemma.

Le mma 2. If $\delta_{\tau, \omega} f_{s}=\tau \tilde{\delta} f_{s+1}+(v-s)(\tilde{\delta} \tau) f_{s+1}, v \geqslant 1$ then $f_{s, v-1}$ will transform independently of $f_{s+1, v}$.

Proof. Examining $(2.1)-(2.4)$, we can see that it suffices to take $\tau=y$. The multipole expansion for $a$ spin $s$ function begins with $2^{|s|}$-pole term, thus, the assertion $\delta f_{s, v-1}=0$ is trivial for $s \geqslant v$, particularly $y \tilde{\delta} f_{v+1, v}=\delta f_{v, v}+\delta f_{v, v+1}$. Acting on the previous equation with $\tilde{\delta}$, we get

$$
y \tilde{\partial} \tilde{f}_{v+1, v}+(\tilde{\partial} y) \tilde{\partial} f_{v+1, v}=\delta \tilde{\delta} f_{v, v}+\delta \tilde{\delta} f_{v, v+1} .
$$

For $l \geqslant|s|$ each $f_{s-1, l}$ depends linearly on $\tilde{\partial}_{s, l}$, hence, we may further write $y \tilde{\delta} f_{v, v}+(\tilde{\partial} y) f_{v, v}=\delta f_{v-1, v}+\delta f_{v-1, v+1}$.

Continuing to act with the $\tilde{\delta}$-operator and using $\tilde{\partial} \tilde{\partial} y=0$, we successively get for each $s>-v$

$$
y \tilde{\delta} f_{s+1, v}+(v-s)(\tilde{\partial} y) f_{s+1, v}=\delta f_{s, v}+\delta f_{s, v+1} .
$$


For $s \leqslant-v$, the result again becomes trivial, as $\delta f_{s, v-1}$ does not exist. The last equation completes the proof of lemma 2.

\section{Appendix D. Variation of physical quantities according to (2.6)}

1. Time translation $(2.4)$ by $\delta t$ :

$$
\delta N=-(1 / 3) \delta t \tilde{\delta}\left(3 p^{k} y_{k}\right)+\ldots, \quad \delta d_{k}=-p^{k} \delta t .
$$

2. Translations (2.3) by $\delta x^{j}$ using (B.9):

$$
\begin{aligned}
\delta N & =\delta x^{j}\left\{-(1 / 3) y_{j} \tilde{\partial}-\left(\tilde{\partial} y_{j}\right)\right\}\left\{m+3 p^{k} y_{k}+\ldots\right\}= \\
& =-\delta x^{j} m \tilde{\partial} y_{j}+\mathrm{i} \varepsilon_{j k l} \delta x^{j} p^{k} \tilde{\partial} y_{l} ; \\
\delta d^{j} & =-\delta x^{j} m, \quad \delta l_{i}=\varepsilon_{i j k} \delta x^{j} p^{k} .
\end{aligned}
$$

3. 3-rotations (2.2) by $\delta \alpha^{k}$ using (B.5):

$$
\delta M=\left(\mathrm{i} \delta \boldsymbol{\alpha}^{j} / 2\right)\left\{\left(\partial y_{j}\right) \tilde{\delta}-\left(\tilde{\partial} y_{j}\right) \delta\right\}\left\{m+3 p^{k} y_{k}+\ldots\right\}=3 \varepsilon_{j k l} \delta \alpha^{j} p^{k} y_{l}+\ldots .
$$

Due to (C.2), (C.1) analogical equation holds for $N$, so we obtain uniformly for $p, d$ and $l$ :

$$
\delta p^{i}=\varepsilon_{i j k} \delta \alpha^{j} p^{k}, \quad \delta d^{i}=\varepsilon_{i j k} \delta \alpha^{j} d^{k}, \quad \delta l^{i}=\varepsilon_{i j k} \delta \alpha^{j} l^{k} .
$$

4. Proper Lorentz transformations (2.1) by $\delta v^{k}$ for $M$ using (C.3)(C.5) and (B.7):

$$
\begin{aligned}
& \delta M=\delta v^{j} \widetilde{R}_{3,0}\left(u y_{j}, \tilde{\partial}_{y_{j}}\right)\left\{m+3 p^{k} y_{k}+\ldots\right\} ; \\
& \delta m=-\delta v^{k} p^{k}, \quad \delta p^{k}=-\delta v^{k} m .
\end{aligned}
$$

5. Proper Lorentz transformations $(2.1)$ by $\delta v^{k}$ for $N$ :

$$
\begin{aligned}
& \delta N=\delta v^{j} \widetilde{R}_{3,-1}\left(u y_{j}, \tilde{\delta} y_{j}\right)\left\{\left(d^{k}-\mathrm{i} l^{k}\right) \tilde{\partial} y_{k}+\ldots\right\}+ \\
& +u \delta v^{j}\left\{-(1 / 3) y_{j} \tilde{\delta}-\left(\tilde{\delta} y_{j}\right)\right\}\left\{m+3 p^{k} y_{k}+\ldots\right\}
\end{aligned}
$$

With (C.3)-(C.5), (B.9) we find

$$
\delta v^{j} \tilde{R}_{3,-1}\left(u y_{j}, \tilde{\partial} y_{j}\right) \tilde{\partial} y_{k}=-\mathrm{i} \varepsilon_{j k l} \delta v^{j} \tilde{\partial} y_{l}
$$

and

$$
\begin{gathered}
\delta v^{j}\left\{-(1 / 3) y_{j} \tilde{\delta}-\left(\tilde{\partial} y_{j}\right)\right\}\left\{m+3 p^{k} y_{k}+\ldots\right\}= \\
=i \varepsilon_{j k l} \delta v^{j} p^{k} \tilde{\partial} y_{l}-m \delta v^{j} \tilde{\partial} y_{j} ;
\end{gathered}
$$

hence,

$$
\delta d^{i}=-\varepsilon_{i j k} \delta v^{j} l^{k}+u m \delta v^{i}, \quad \delta l^{i}=\varepsilon_{i j k} \delta v^{j}\left(d^{k}-u p^{k}\right) .
$$

\section{REFERENCES}

1. Bondi, H. Burg, M. G. J. van der, Metzner, A. W. K. Proc. Roy. Soc., A269, №1336, $21-52$ (1962).

2. Sachs, R. Phys. Rev., 128, № 6, 2851-2864 (1962).

3. Tod, K. P. Classical and Quantum Gravity, 3, № 6, 1169-1189 (1986).

4. McCarthy, P. J. Proc. Roy. Soc., A330, № 1583, 517-535 (1972).

5. Unt, 4. Proc. Acad. Sci. ESSR. Phys. Math., 36, № 1, 14-20 (1987).

6. Kuusk, P., Unt, V. Gen Relativity and Gravitation, 7, № 5, 399-417 (1976). 
A. UNT

\section{FUUSIKALISTE SUURUSTE VARIATSIOONID BMS-RÜHMA TÉISENDUSTEL}

Lähtudes kompaktselt kirjapandud Bondi integreerimisfunktsioonide teisenemiseeskirjast (1.5) ja täiendavatest seostest (1.8), on leitud, mil määral massi, impulsi, pöördimpulsi ja dipoolmomendi teisenemine Poincaré rühma mõjul vastab eeldatavale, ja selgitatud, kuidas mõista pöördimpulsi teisenemist supertranslatsioonidel.

\section{A. УHT}

\section{ВАРИАЦИИ ФИЗИЧЕСКИХ ВЕЛИЧИН ПРИ БМС-ПРЕОБРАЗОВАНИЯХ}

Исходя из правила преобразования функций интегрирования Бонди (1.5) и из дополнительных уравнений (1.8) для них, во-первых, найдено в какой мере преобразования массы, импульса, импульса вращения и дипольного момента соответствуют ожидаемым, и, во-вторых, выяснено, как понять преобразование импульса вращения при супертрансляциях. 